\title{
Operationalizing a proposed national protocol for the prevention and management of severe pre-eclampsia and eclampsia using a loading dose of magnesium sulphate at community health facilities in Bangladesh
}

Population Council

Follow this and additional works at: https://knowledgecommons.popcouncil.org/departments_sbsr-rh

Part of the Community-Based Research Commons, Demography, Population, and Ecology Commons, International Public Health Commons, and the Maternal and Child Health Commons How does access to this work benefit you? Let us know!

\section{Recommended Citation}

"Operationalizing a proposed national protocol for the prevention and management of severe preeclampsia and eclampsia using a loading dose of magnesium sulphate at community health facilities in Bangladesh." Washington, DC: Population Council, 2014. 
OPERATIONALIZING A PROPOSED NATIONAL PROTOCOL FOR THE PREVENTION AND MANAGEMENT OF SEVERE PRE-ECLAMPSIA AND ECLAMPSIA USING A LOADING DOSE OF MAGNESIUM SULPHATE AT COMMUNITY HEALTH FACILITIES IN BANGLADESH 


\section{Table of Contents}

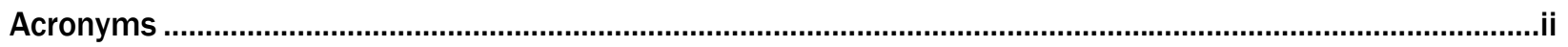

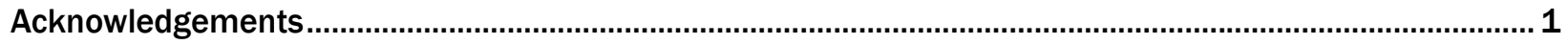

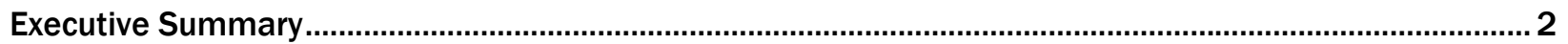

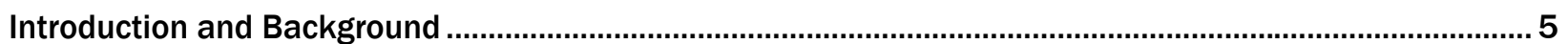

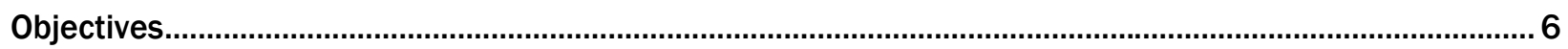

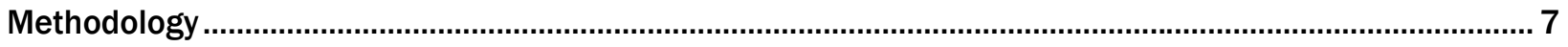

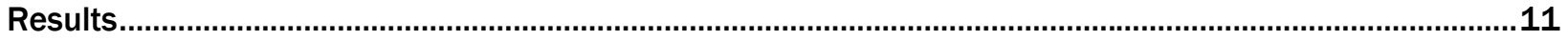

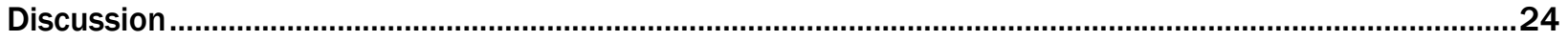

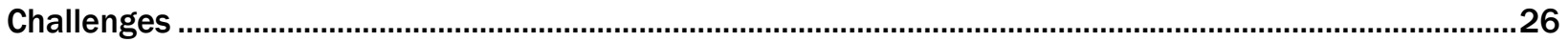

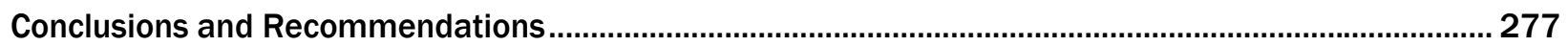

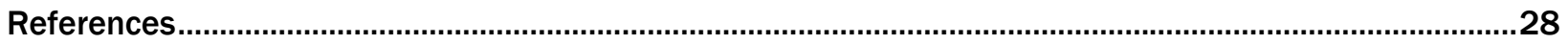




\section{Acronyms}

\begin{tabular}{|c|c|}
\hline ANC & Antenatal Care \\
\hline BCC & Behavioral Change Communication \\
\hline BDHS & Bangladesh Demographic and Health Survey \\
\hline BMMS & Bangladesh Maternal Mortality Survey \\
\hline $\mathrm{CHCP}$ & Community Health Care Provider \\
\hline CS & Civil Surgeon \\
\hline CSBA & Community Skilled Birth Attendant \\
\hline DDFP & Deputy Director of Family Planning \\
\hline DGFP & Directorate General of Family Planning \\
\hline DGHS & Directorate General of Health Service \\
\hline EmOC & Emergency Obstetric Care \\
\hline FP & Family Planning \\
\hline FPI & Family Planning Inspector \\
\hline FWA & Family Welfare Assistant \\
\hline FWV & Family Welfare Visitor \\
\hline HA & Health Assistant \\
\hline IEC & Information, Education and Communication \\
\hline IM & Intramuscular \\
\hline $\mathrm{IMCl}$ & Integrated Management of Childhood Illness \\
\hline MA & Medical Assistant \\
\hline MCWC & Maternal and Child Welfare Center \\
\hline MDG & Millennium Development Goal \\
\hline $\mathrm{MgSO}_{4}$ & Magnesium Sulphate \\
\hline MMR & Maternal Mortality Rate \\
\hline MO & Medical Officer \\
\hline MoH\&FW & Ministry of Health and Family Welfare \\
\hline MO-MCHFP & Medical Officer of Maternal and Child Health and Family Planning \\
\hline MoU & Memorandum of Understanding \\
\hline NGO & Non-Governmental Organization \\
\hline OGSB & Obstetrical and Gynecological Society of Bangladesh \\
\hline OR & Operations Research \\
\hline PE & Pre-Eclampsia \\
\hline PNC & Postnatal Care \\
\hline RMO & Resident Medical Officer \\
\hline SACMO & Sub-Assistant Community Medical Officer \\
\hline SPE & Severe Pre-Eclampsia \\
\hline SPSS & Statistical Package for the Social Sciences \\
\hline TA & Technical Assistance \\
\hline UFPO & Upazila Family Planning Officer \\
\hline UH\&FWC & Union Health and Family Welfare Center \\
\hline UHC & Upazila Health Complex \\
\hline USAID & United States Agency for International Development \\
\hline
\end{tabular}




\section{Acknowledgements}

The Population Council and the Obstetrical and Gynecological Society of Bangladesh thank the John D. and Catherine T. MacArthur Foundation for their financial support for this study, in conjunction with the Population Council.

We are thankful to Dr. Mohammad Sharif, Director of Maternal and Child Health and Line Director for Maternal, Child, Reproductive and Adolescent Health of the Directorate General of Family Planning, and Dr. Abu Jafar Md. Musa, Director of Primary Health Care and Line Director for Maternal, Newborn, Child and Adolescent Health of the Directorate General of Health Science, for their overall guidance in implementing project activities.

We would like to thank Dr. Abu Jamil Faisel and Dr. Farhana Akter of EngenderHealth for their continued technical support in all aspects of this study's implementation.

Our sincere gratitude to the members of the technical committee Dr. Latifa Shamsuddin, President of the Obstetrical and Gynecological Society of Bangladesh, Dr. Parveen Fatima, Secretary General of the Obstetrical and Gynecological Society of Bangladesh, Dr. Ubaidur Rob, Country Director of the Population Council, and Dr. Sharif Mohammed Ismail Hossain, Associate of the Population Council, for their continuous support and cooperation in planning and implementing this study.

We are grateful to Dr. Farhana Khan and Dr. Fowzia Akhter, Junior Consultants for Obstetrics and Gynecology, Brahmanbaria District Hospital, for their continuous support as a resource persons and mentors for patient referral management.

Last, but not least, we are indebted to the Civil Surgeon and Deputy Director of Family Planning of Brahmanbaria district, as well as Union Health and Upazila Family Planning Officers, Resident Medical Officers, Medical Officers of Maternal and Child Health and Family Planning, Upazila Family Planning Officers, other consultants, medical officers, and all community facility-based service providers and field workers in Sarail and Kasba upazilas of Brahmanbaria district for their support, cooperation, and hard work during implementation of this study. 


\section{Executive Summary}

About 20 percent of maternal deaths in Bangladesh are caused by pre-eclampsia and eclampsia. Most of these deaths occur during care within local communities due to lack of maternal health information, poor antenatal care attendance, and delays in seeking care. Most of these deaths can be easily prevented with low cost maternal health commodities. It is proposed that if local community facility service providers are trained to detect pre-eclampsia and eclampsia, administer a loading dose of magnesium sulphate, and refer patients within time to a referral facility or physician, perinatal mortality will be greatly reduced. Bangladesh's Ministry of Health and Family Welfare has shown keen interest in a recently developed protocol implementing this proposed methodology, within its current program structure, by training service providers based at local community or primary health care facilities.

A quasi-experimental pre- and post-test study, with no control group, was implemented to assess the abilities of community facility service providers to screen and detect eclampsia patients, administer a loading dose of magnesium sulphate to diagnosed patients, and refer them to an appropriate facility for condition management. These interventions were instituted for nine months in 19 Union Health and Family Welfare Centers and 53 community clinics in two upazilas in Brahmanbaria district. This study was conducted in operational public health clinics without any major health system changes. Government health and family planning service providers based at community health care facilities implemented the treatment protocol, and government field workers conducted awareness activities and identified high risk pregnant women, to refer them to Union Health and Family Welfare Centers.

This study trained primary health care providers to screen and detect pre-eclampsia and eclampsia, and administer a loading dose of magnesium sulphate to prevent and control convulsions and seizures. Field workers were provided with necessary information on pre-eclampsia and eclampsia to educate pregnant women of their risks, identify high risk pregnant women, and refer them to local health care facilities. In addition, operational links between primary care and referral facilities were established.

Findings suggest significant improvements in provider knowledge of pre-eclampsia, severe pre-eclampsia, and eclampsia following the intervention period. Knowledge of high blood pressure as a sign of preeclampsia improved from 60 percent to 91 percent post-intervention. Similarly, knowledge of danger signs during pregnancy increased substantially after the intervention, including knowledge of seizures and convulsions as danger signs (from $87 \%$ to 94\%). Service provider self-assessments suggest that all are able to measure blood pressure, test urine for albumin, and administer a loading dose of magnesium sulphate intramuscular injection before referring patients with sufficient time for case management.

Service statistics suggest that numbers of patients, for antenatal, delivery, and postnatal care services, did not change over the intervention period; however, political unrest in the country during the first three months of the study-one third of its duration-may have affected patient attendance. Prior to the intervention there were no documented pre-eclampsia and eclampsia patients in the two intervention upazilas, but during the intervention period 33 patients with severe pre-eclampsia and eclampsia sought services at local public health facilities. More than two thirds of patients suffered from seizures, while the rest presented with severe pre-eclampsia: high blood pressure, proteinuria, and other signs or symptoms.

District findings suggest that most pre-eclampsia and eclampsia patients seek services directly from the district hospital, rather than first presenting at local or community health care facilities. Primary health care providers suggest that women choose services from district hospitals due to ease of transportation to them $(81.8 \%)$, or for specialist care $(71.7 \%)$, and lack of confidence in their local community facilities. Program managers, however, believe women go to district hospitals due to lack of available health care providers at primary health care facilities, perceptions of primary care providers' abilities for managing pre-eclampsia 
and eclampsia patients, as well as their lack of confidence in local facility staff capacities for managing emergency conditions including as eclampsia.

During this intervention all patients with severe pre-eclampsia and eclampsia were treated by Family Welfare Visitors at Union Health and Welfare Centers.

Although other community facility-based health care workers such as Sub-Assistant Community Medical Officers, Medical Assistants, Community Health Care Providers, and Community Skilled Birth Attendants were trained and included in this eclampsia service provision protocol, none of them actually treated any pre-eclampsia and eclampsia patients during the nine month implementation, providing no means of assessing their capabilities for providing pre-eclampsia and eclampsia services.

There were no maternal or neonatal deaths among those who received a magnesium sulphate intramuscular injection at a community facility-however one infant died at a referral facility. No serious adverse event due to loading dose of magnesium sulphate intramuscular injection was reported in the study population.

As only Family Welfare Visitors initially treated women with pre-eclampsia and eclampsia at community health care facilities, it suggests that only Family Welfare Visitors should be incorporated within the national protocol for detecting, preventing, and managing pre-eclampsia and eclampsia within local community care systems.

\section{Conclusions and Recommendations}

Most eclamptic women reported seizures as their primary complaint, while women with severe preeclampsia reported variable high blood pressure, proteinuria, and other signs or symptoms. Those service providers who examined women with severe pre-eclampsia and eclampsia administered a loading dose of magnesium sulphate intramuscular injection and referred them with sufficient time for appropriate case management. Women with severe pre-eclampsia and eclampsia did visit local Union Health and Family Welfare Centers, but none went to a community clinic or contacted a Community Skilled Birth Attendant.

All service providers involved in the study, as well as their program managers, are enthusiastic about the intervention and suggested expanding it to other parts of the country. Replicating this intervention in a phased manner is strongly advised, first in a larger area to further understand operational details and complexities, to determine if any adjustments are necessary before expansion throughout the country. The unit cost for scaling up the intervention per upazila or sub-district is calculated as US $\$ 7,948$, with an annual recurring cost of US $\$ 2,052$. Refresher trainings will cost US $\$ 4,046$ per upazila.

It is recommended that the intervention be fully implemented only through Family Welfare Visitors, and potentially Sub-Assistant Community Medical Officers, with a new national protocol for community management of pre-eclampsia and eclampsia, or the adaptation of the present protocol, with simplified procedures. Satellite clinics are an awkward and inappropriate place for this intervention, particularly urine testing, which demands a washroom.

Community health care providers require mentorship, especially at the beginning of the intervention. Referral facilities must each identify a resource person to develop an effective upazila referral system. Local and community service providers should have direct access to their referral resource personnel, via mobile phone, for assistance when needed.

Strategies need to be developed and implemented to ensure availability of services and service providers at community facilities. Monitoring and supervision is crucial in this regard. Collaboration is needed between both Ministry of Health and Family Welfare directorates to make the program successful. 
Field workers did not refer any clients with signs and symptoms consistent with pre-eclampsia and eclampsia, instead patients sought emergency treatment. To make the program sustainable, field workers such as Family Welfare Assistants and Health Assistants should be fully motivated and involved to raise community awareness of pre-eclampsia and eclampsia, in addition to detecting pre-eclampsia and eclampsia patients' signs and symptoms, and referring them to their community health care facilities, if needed.

More than half of the community service providers evaluated their training for this study intervention as inadequate. Further training should include broader maternal health issues including antenatal, delivery, and postnatal care, not only pre-eclampsia and eclampsia. Service providers want refresher training, and it should be included in the service protocol, after six months of implementation, to refresh provider knowledge, share experiences, and offer solutions, if any. The general primary health care provider educational curriculum needs to be reviewed for full incorporation of pre-eclampsia and eclampsia diagnosis and treatment.

It is preferable that the solution of magnesium sulphate intramuscular injection for a loading dose should be in one or two vials instead of four vials. Liaison and advocacy with manufactures is urgently required. Essential equipment such as blood pressure machines and stethoscopes are either non-functioning or not available at community facilities. Test tubes for urine collection and testing, and disposable syringes and needles, are also not available at community facilities. All of these materials are required to implement this protocol.

As community health care facilities lack the people's confidence, generally, these facilities should be given renewed attention by health system authorities, for renewal and rebranding so that their catchment populations are more confident in the care provided by these facilities, in addition to ensuring that community facility service providers are regularly available, according to a set schedule. In addition, awareness campaigns, through local field workers as well as local government structures and available media, need to be developed and implemented on the availability of community pre-eclampsia and eclampsia detection, immediate treatment, and referral services. 


\section{Introduction and Background}

Hypertensive disorders during pregnancy are an important cause of morbidity, long term disability, and death among mothers and their infants. About 10 percent of women have increased blood pressure (BP) during pregnancy, and two to eight percent of pregnancies are complicated by pre-eclampsia or eclampsia (PE/E). About 10 percent of pre-eclampsia patients develop severe PE/E (SPE/E). Globally, about 10 to 20 percent of maternal deaths are associated with eclampsia ${ }^{1}$. Most deaths due to SPE/E are preventable with timely and effective medical care².

The 2010 Bangladesh Maternal Mortality Survey (BMMS 2010) suggests that postpartum hemorrhage, severe $\mathrm{PE} / \mathrm{E}$, and prolonged and obstructed labor are the major three causes of maternal mortality, while indirect causes contribute about 35 percent of maternal deaths in Bangladesh. Maternal deaths decreased substantially, from 322 in 2001 to 194 in 2010, per 100,000 live births, a 40 percent decline in nine years, but the country's maternal mortality rate (MMR) remains high. Bangladesh has one of the highest MMRs in South Asia, with about 7,000 to 8,000 women dying every year from pregnancy complications ${ }^{3}$. Bangladesh has to reduce its MMR to 143 per 100,000 live births by 2015 to achieve MDGs 4 and 5 .

No specific study in Bangladesh has investigated PE/E incidence and prevalence rates except the BMMS, which records SPE/E as significant cause of maternal death. A survey of emergency obstetric care (EmOC) services in Bangladesh in 1995 revealed a national incidence of convulsion in pregnancy and puerperium of approximately eight percent, ${ }^{5}$ of which a large number was likely due to eclampsia. BMMS 2010 revealed eclampsia to be the second most significant direct obstetric cause of maternal death in Bangladesh, contributing to 20 percent of all maternal deaths ${ }^{3}$.

Preliminary data from the 2011 Bangladesh Demographic and Health Survey (BDHS) show that 68 percent of women received antenatal care (ANC) at least once. Most women (55\%) received care from a medicallytrained provider. Comparable data from the 2004 and 2007 BDHS show increases in ANC from a service provider, of 17 percent (from 58\% in 2004 to 68\% in 2011), ANC from a medically-trained provider during the same period increased only by four percent (from $51 \%$ to $55 \%$, respectively) ${ }^{6}$. Seventy-one percent of women delivered at home, due to illiteracy, preference for home delivery, poverty, fear for caesarean section, and superstition. As a result, most pre-eclampsia cases remain unrecognized until severe complications occur.

Bangladesh's health and family planning (FP) system is fairly well established in rural areas. Urban areas are mainly served by tertiary medical institutions and private clinics. District hospitals and Maternal and Child Welfare Centers (MCWCs) are found in all 64 districts, serving populations of 1.5 to 2.0 million each, while 484 Upazila Health Complexes (UHCs) of upazilas or sub-districts serve 0.25 to 0.35 million people each, and approximately 4,000 Union Health and Family Welfare Centers (UH\&FWCs) and rural dispensaries (RDs) each serve 25,000 to 35,000 people. (Most RDs have now been converted to UH\&FWCs.) Community clinics (CCs), an initiative of the current government, are designed to provide health care to rural populations of about 6,000 .

District hospitals provide all types of curative health services, while MCWCs provide FP and maternal health services including ANC, normal delivery and caesarian sections, and postnatal care (PNC). UHCs provide curative health and FP services including ANC, normal delivery, PNC, vaccinations, health education, in addition to child care. UH\&FWCs provide health and FP services including child and maternal health care. Every year each UH\&FWC features approximately 500 to 600 infant deliveries, with one maternal death on average. About 30,000 satellite clinics every month provide the same health and FP services as UH\&FWCs, while CCs provide health and FP services within the smallest communities. 
Various categories of service providers-Medical Officers (MOs), Family Welfare Visitors (FWVs), Medical Assistants (MAs), Sub-Assistant Community Medical Officers (SACMOs), and Community Health Care Providers (CHCPs) - offer health care at UH\&FWCs, RDs, and CCs, and are supported by field workers-either Family Welfare Assistants (FWAs) or Health Assistants (HAs).

All FWVs are female, with 18 months basic training in reproductive health $(\mathrm{RH})$ and child health care. They provide basic FP and maternal and child health $(\mathrm{MCH})$ services. SACMOs have three years of basic health training and are responsible for general health care and treatment of minor ailments in local communities. A cadre of FWAs trained in midwifery (for six months), called Community Skilled Birth Attendants (CSBAs), are posted in communities for delivery care. CHCPs, who staff CCs, receive six months of training to provide basic health, FP, and immunization services.

Due to maternal deaths from PE/SPE/E, the Ministry of Health and Family Welfare (MoH\&FW) has implemented various initiatives to reduce these preventable deaths. The Eclampsia Collaborative Trial ${ }^{7}$ and Magpie studies ${ }^{8}$ confirmed the efficacy of magnesium sulphate $\left(\mathrm{MgSO}_{4}\right)$ in treating severe $\mathrm{PE} / \mathrm{E} . \mathrm{MgSO}_{4}$ is available at secondary and tertiary hospitals for treatment of SPE/E, and trained providers are mandated to administer it. Several pharmaceutical companies produce $\mathrm{MgSO}_{4}$, and it is available throughout the country, but the extent of its use is unknown, as it is officially restricted to use in higher level facilities.

To prevent PE/E deaths and morbidities, MoH\&FW developed a protocol for the administration of a loading dose of $\mathrm{MgSO}_{4}$ at community health care centers, by FWVs, SACMOs, nurses, CSBAs, and CHCPs. The protocol was finalized in 2014, with technical assistance (TA) from the Obstetrical and Gynecological Society of Bangladesh (OGSB) and EngenderHealth (Appendix 1). MoH\&FW has shown keen interest in implementing the protocol, and is eager to understand the operational complexities of its introduction.

In collaboration with the directorates general of Health Services (DGHS) and Family Planning (DGFP), OGSB implemented the strategies of the proposed national protocol with support from the Population Council, in a study to assess the efficacy of screening and detecting SPE/E and providing a loading dose of $\mathrm{MgSO}_{4}$ at community facilities, by FWVs, SACMOs, nurses, CSBAs, and CHCPs.

\section{Objectives}

\section{General Objective}

The general objective of this study is to assess the abilities of community facility-based service providers for screening and detecting PE/E patients, then administering a loading dose of $\mathrm{MgSO}_{4}$ to appropriate patients and referring them to higher level care facilities.

\section{Specific Objectives}

The specific objectives are:

- Assessing the abilities of service providers-FWVs, SACMOs, nurses, CSBAs, CHCPs-based at community health care facilities to screen and detect PE/E within their local health care networks

- Assessing local service providers' abilities to provide a loading intramuscular (IM) dose of $\mathrm{MgSO}_{4}$

- Reviewing the provision of $\mathrm{MgSO}_{4}$ loading doses at local community care facilities and their referrals of patients for to higher level facilities for appropriate case management

- Assessing the effectiveness of the referral mechanisms, and

- Analyzing the costs of these interventions. 


\section{Methodology}

\section{Study Design}

This quasi-experimental study, of a pre-test post-test design with no control group, implemented interventions for nine months in two upazilas. Before implementation, service statistics (numbers of ANC, delivery and PNC clients, hypertensive pregnant clients, and PE/E patients) from both community and referral facilities were collected. Service provider and field worker knowledge of PE/E was also evaluated. At the end of intervention, the procedures for the baseline were repeated to collect endline information. Comparisons of data from before and after the intervention measure the impacts of the intervention.

This study was implemented and managed by OGSB in collaboration with DGHS and DGFP, with TA by the Population Council and EngenderHealth. Ethics board approval for conducting the study was obtained from the Bangladesh Medical Research Council (BMRC) and Population Council's Institutional Review Board.

\section{Study Location}

The study was implemented in Sarail and Kasba upazilas of Brahmanbaria district. There are nine UH\&FWCs and 21 CCs in Sarail, and 10 UH\&FWCs and 32 CCs in Kasba Upazila, with 72 community facilities in total in the two upazilas selected to test the national protocol. Two UHCs and the Brahmanbaria district hospital and MCWC were the referral facilities.

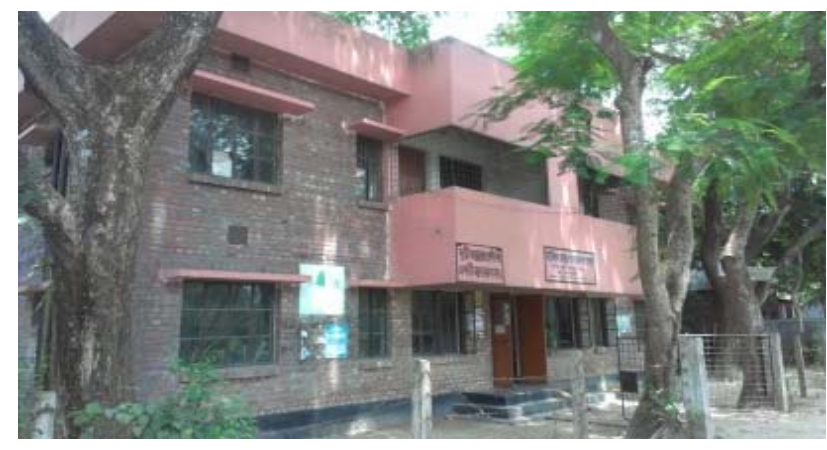

Union Health and Family Welfare Center Community Health Care Facility

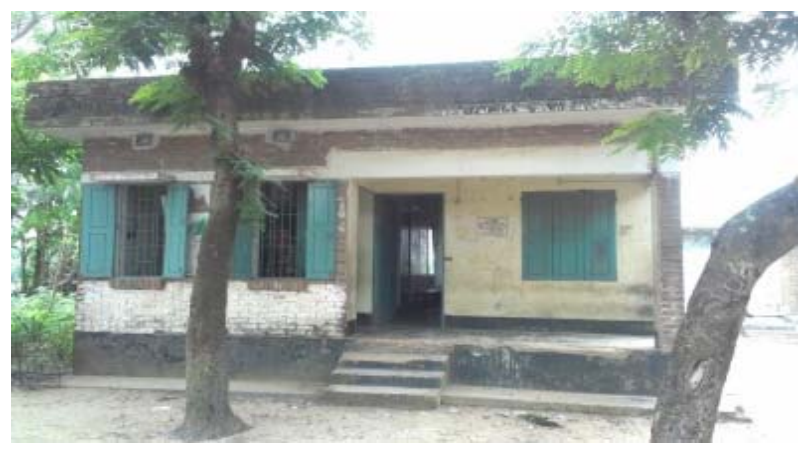

Community Clinic

\section{Study Period}

The study was completed in 14 months, from July 2013 through July 2014. The actual protocol was implemented from October 2013 through June 2014. An initial three months involved preparatory work and the baseline data collection, and the final two months involved the endline evaluation, report writing, and dissemination.

\section{Study Population}

The primary population of this study are health care service providers, including FWVs, nurses, SACMOs, CHCPs, and CSBAs, working at local community facilities, either UH\&FWCs or CCs.

This study's secondary population of interest is pregnant women with high blood pressure (BP), especially $\mathrm{PE} / \mathrm{E}$ patients, in addition to upazila and district health care managers and service providers, in addition to the field workers who support local community facility service providers. 


\section{Intervention}

The following interventions were implemented in the selected upazilas:

Training for PE/E screening and detection among pregnant women by community facility service providers: A total of 140 service providers (FWVs, SACMOs, nurses, CSBAs. and CHCPs) posted at Sarail and Kasba upazilas attended training on $\mathrm{PE} / \mathrm{E}$ detection and management, measuring $\mathrm{BP}$, urine dipstick test, administering $\mathrm{MgSO}_{4}$ loading dose, and referral. Resource persons were CS, DDFP, junior obstetrics and gynecology consultants from Brahmanbaria District Hospital, and staff from OGSB, EngenderHealth, and Population Council.

Orienting field workers to educate pregnant women, identify high risk pregnant women, and refer them to service providers at community facilities: A total of 163 field workers (FWAs and HAs) participated in the upazila orientations, and were informed about the project objectives: detecting $\mathrm{PE} / \mathrm{E}$ during home care visits, raising $\mathrm{PE} / \mathrm{E}$ awareness within their communities, and referring potential $\mathrm{PE} / \mathrm{E}$ patients to community facilities for further management. Orientations were by OGSB, with representatives from DGHS and DGFP.

Orienting upazila and district program managers and service providers: A total of 16 UH\&FPOs, UFPOs, doctors, consultants, FPIs, HIs, and AHIs participated in the district orientation program on PE/E management and their roles in the interventions, led by OGSB, EngenderHealth, Population Council, and representatives from DGHS and DGFP.

Developing educational and training materials: A client IEC material was developed and distributed within study communities. EngenderHealth and OGSB developed the training manual, job aids, and leaflets used during the intervention.

Establishing functional referral systems between service providers at community facilities and higher level facilities, with referral facility staff orientation on the project, its objectives, and interventions. Referral facility staff were trained on the referral slip to be completed by community service providers. Referral facility service providers then trained community facility service providers.

Procuring required equipment, logistics, and supplies for community facilities: 69 BP machines, 69 stethoscopes, 60 boxes of urine dipsticks, and 3,800 ampoules of $\mathrm{MgSO}_{4}$.

Administering a loading dose of $\mathrm{MgSO}_{4}$ to prevent and control convulsions and seizures by trained service providers to patients who sought treatment at community health care facilities for $\mathrm{PE} / \mathrm{E}$.

\section{Study Implementation}

The study was conducted in operational government health care facilities, without any significant system alterations. Government health and FP service providers who work at community facilities implemented the program, with field workers conducting awareness activities, identifying high risk pregnant women, and referring them to community UH\&FWC facilities. OGSB implemented the intervention in collaboration with DGFP and DGHS, with TA from the Population Council and EngenderHealth.

\section{Field Supervision and Monitoring}

In addition to regular monitoring of the local public health care systems, project staff regularly monitored field activities. Community health care facilities in the intervention upazilas were visited to monitor service provision by community service providers. Project staff attended Health and FP department monthly meetings of community service providers in both Sarail and Kasba upazilas, during which PE/E was included on each meeting's agenda, followed by a short discussion. 


\section{Evaluation}

The effects of the intervention were assessed by evaluating community service provider abilities to detect and manage $\mathrm{PE} / \mathrm{E}$ patients including administration of $\mathrm{MgSO}_{4}$. Surveys compared $\mathrm{PE} / \mathrm{E}$ service statistics pre- and post intervention and improvements in PE/E knowledge among community service providers, in addition to self-administered checklists for providers, comparisons of patient care quality through exit interviews with a semi-structure questionnaire, and observations of client and provider interactions, using a checklist. Program managers and referral service providers were also interviewed before and after the intervention, with interview guides, to assess staff opinions about the project interventions and community facility service providers' responsibility for administering a loading dose of $\mathrm{MgSO}_{4}$ and referring patients.

Table 1: Type of data collection, number, timing and contents

\begin{tabular}{|l|c|c|l|}
\hline \multirow{2}{*}{$\begin{array}{l}\text { Evaluation } \\
\text { Component }\end{array}$} & \multicolumn{2}{|c|}{$\begin{array}{c}\text { Frequency and Number } \\
\text { of Respondents }\end{array}$} & \\
\cline { 2 - 4 } & $\begin{array}{c}\text { Pre- } \\
\text { Intervention }\end{array}$ & $\begin{array}{c}\text { Post- } \\
\text { Intervention }\end{array}$ & \\
\hline $\begin{array}{l}\text { Service Provider } \\
\text { Interviews (CLFBSP) }\end{array}$ & 105 & 105 & $\begin{array}{l}\text { PE/E knowledge, attitude and practices for detecting and managing, } \\
\text { efficiency of ANC, PNC and delivery services }\end{array}$ \\
\hline $\begin{array}{l}\text { Field Worker } \\
\text { Interviews }\end{array}$ & 174 & 50 & $\begin{array}{l}\text { PE/E knowledge, attitudes and opinions, ability to detect cases } \\
\text { within communities, and refer }\end{array}$ \\
\hline $\begin{array}{l}\text { Client Exit Interviews } \\
\text { Client and Provider } \\
\text { Observations }\end{array}$ & 40 & 39 & Quality of services for ANC, PNC, and delivery clients \\
\hline $\begin{array}{l}\text { Program Manager } \\
\text { and Referral Service } \\
\text { Provider Interviews }\end{array}$ & 14 & 39 & Quality of services for ANC, PNC, and delivery clients \\
\hline Service Statistics & $\begin{array}{c}\text { Preceding } \\
9 \text { months }\end{array}$ & $\begin{array}{c}\text { Intervention } \\
(9 \text { months) }\end{array}$ & $\begin{array}{l}\text { Number of ANC, PNC, delivery, and PE/E clients } \\
\text { in integrating PE/E into community facilities }\end{array}$ \\
\hline
\end{tabular}

Trained research assistants collected the baseline and endline data. The same individuals collected the baseline and endline data. Baseline data were collected in September and October 2013, and endline data were collected in May and June 2014.

\section{Data Analysis}

Quantitative data were collected from service providers, field workers, clients after exiting service sessions at health facilities, and observations of client and provider interactions, and were analyzed using SPSS. Findings are presented in the Results section in a descriptive manner, with univariate and bivariate frequency tables, segregated as pre- and post. Qualitative data were collected from managers and service providers, and were analyzed manually according to themes, and triangulated with quantitative findings.

\section{Limitations}

The study had several limitations:

- Observations of actual PE/E service provision by the community facility service providers were not possible due to uncertainty of when clients would present at a facility. This study relied upon service providers' self-assessments of their administrations of $\mathrm{MgSO}_{4} \mathrm{IM}$ injection loading dose. All service providers were trained in IM injection, and the study employed a $\mathrm{MgSO}_{4}$ solution.

- Although each facility was given a project register to record ANC, PE/E, delivery, and PNC patient data, service providers did not fully comply with recording requests, so MIS reporting statistics submitted to DGFP were collected for quantitative data for this report. 
- Community facility service provider training was limited to detecting, by physical examination, BP measurement, and urine test, $\mathrm{PE} / \mathrm{E}$ signs and symptoms, its management, administration of a loading dose of $\mathrm{MgSO}_{4}$, and patient referrals. The project was unable to provide training on general maternal health, pregnancy complications, delivery issues, postpartum hemorrhage, and PNC complications. Analysis of this project's data suggest that some of these issues need to be addressed during training. 


\section{Results}

Community facility-based service providers' background characteristics were collected during the baseline survey, and the same cohort was interviewed at the endline survey. Table 2 indicates that 19 FWVs, 11 SACMOs, two nurses, 38 CSBAs, and 35 CHCPs were interviewed in the two upazilas. All FWVs, nurses, and CSBAs were female, all SACMOs were male, with eight male and 27 female CHCPs.

Table 2: Community facility service providers, by background characteristics

\begin{tabular}{|c|c|c|c|c|c|c|}
\hline \multirow{2}{*}{$\begin{array}{l}\text { Characteristics } \\
\text { Sex }\end{array}$} & \multicolumn{5}{|c|}{ Community facility service providers } & \multirow[t]{2}{*}{ Total } \\
\hline & FWV & SACMO & Nurse & CSBA & $\mathrm{CHCP}$ & \\
\hline Male & 0 & 11 & 0 & 0 & 8 & 19 \\
\hline Female & 19 & 0 & 2 & 38 & 27 & 86 \\
\hline \multicolumn{7}{|l|}{ Age } \\
\hline $20-25$ & 0 & 0 & 0 & 5 & 13 & 18 \\
\hline $26-30$ & 3 & 3 & 0 & 2 & 17 & 25 \\
\hline $31-35$ & 1 & 1 & 2 & 2 & 5 & 11 \\
\hline $36-40$ & 0 & 0 & 0 & 4 & 0 & 4 \\
\hline 41-45 & 6 & 0 & 0 & 11 & 0 & 17 \\
\hline $46-50$ & 3 & 1 & 0 & 12 & 0 & 16 \\
\hline$>50$ & 6 & 6 & 0 & 2 & 0 & 14 \\
\hline \multicolumn{7}{|l|}{ Residence } \\
\hline Inside the facility & 7 & 4 & 2 & 0 & 0 & 13 \\
\hline Outside the facility & 12 & 7 & 0 & 38 & 35 & 92 \\
\hline \multicolumn{7}{|l|}{ Duration of job (in years) } \\
\hline $0-5$ & 4 & 4 & 2 & 9 & 35 & 54 \\
\hline $11-20$ & 1 & 0 & 0 & 1 & 0 & 2 \\
\hline $21-30$ & 11 & 6 & 0 & 27 & 0 & 44 \\
\hline$>30$ & 3 & 1 & 0 & 1 & 0 & 5 \\
\hline \multicolumn{7}{|l|}{ Began work at facility (years prior) } \\
\hline $0-5$ & 12 & 4 & 2 & 12 & 35 & 65 \\
\hline $6-10$ & 1 & 3 & 0 & 1 & 0 & 5 \\
\hline $11-20$ & 4 & 1 & 0 & 6 & 0 & 11 \\
\hline $21-30$ & 2 & 3 & 0 & 18 & 0 & 23 \\
\hline$>30$ & 0 & 0 & 0 & 1 & 0 & 1 \\
\hline \multicolumn{7}{|l|}{ Training in last 3 years* } \\
\hline EmOC & 2 & 0 & 1 & 1 & 1 & 5 \\
\hline ANC & 2 & 1 & 1 & 2 & 15 & 21 \\
\hline Safe delivery care & 3 & 0 & 1 & 1 & 7 & 12 \\
\hline PNC & 2 & 1 & 1 & 4 & 13 & 21 \\
\hline CSBA training & 0 & 0 & 0 & 14 & 0 & 14 \\
\hline FP & 2 & 0 & 0 & 3 & 9 & 14 \\
\hline Laboratory investigation & 1 & 0 & 1 & 0 & 0 & 2 \\
\hline CHCP training & 0 & 0 & 0 & 1 & 8 & 9 \\
\hline $\mathrm{IMCl}$ training & 0 & 1 & 0 & 0 & 0 & 1 \\
\hline Midwifery & 1 & 0 & 0 & 1 & 0 & 2 \\
\hline Child growth & 2 & 0 & 0 & 12 & 3 & 17 \\
\hline Others & 1 & 2 & 1 & 0 & 5 & 6 \\
\hline *Multiple answers. No MA was available & 19 & 11 & 2 & 38 & 35 & 105 \\
\hline
\end{tabular}


FWVs, nurses, CSBAs and SACMOs are likely older than CHCPs, a new service cadre, in addition to longer work experience. Most service providers have been based at their facility for five years of less, but some FWVs, SACMOs, and CSBAs have been at the same facility for over 20 years. FWVs, SACMOs, and nurses are expected to reside at their facilities, but about half of FWVs and more than half of SACMOs do not.

All community facility service providers had received training within the past three years. Almost all received $\mathrm{PE} / \mathrm{E}$ training from the program, but some CHCPs recruited after training did not. Only half of community service providers were trained in ANC, delivery, PNC, or emergency obstetric care (EmOC) within three years.

\section{Community facility service provider knowledge of potential life-threatening complications during ANC, delivery, and PNC}

Facility-based service providers in local communities were interviewed to assess their knowledge of general life-threatening complications during the antenatal, delivery, and postnatal periods, both before their training and after the intervention. In each survey, a total of 105 respondents participated.

Service providers were aware of several complications, and comparisons of baseline and endline findings show some improvements in required knowledge, but for some service providers, knowledge actually decreased. Baseline knowledge of life-threatening antenatal complications was generally high, so it improved only marginally. Two thirds of respondents at baseline mentioned eclampsia or seizure as an antenatal complication, which increased to 69 percent at endline. Similarly, providers mentioning severe headache as a complication during antenatal period increased from 71 percent to 87 percent by the endline survey. Several misconceptions also persisted among service providers. Findings indicate ample room for improving service providers' knowledge further (Table 3).

Table 3: Life-threatening antenatal, delivery and postnatal complications observed by providers*

\begin{tabular}{|l|c|c|c|c|c|c|}
\hline \multirow{2}{*}{ Complications } & \multicolumn{2}{|c|}{ Antenatal (\%) } & \multicolumn{2}{c|}{ Delivery (\%) } & \multicolumn{2}{c|}{ Postnatal (\%) } \\
\cline { 2 - 8 } & $\begin{array}{c}\text { Pre- } \\
\text { Intervention }\end{array}$ & $\begin{array}{c}\text { Post- } \\
\text { Intervention }\end{array}$ & $\begin{array}{c}\text { Pre- } \\
\text { Intervention }\end{array}$ & $\begin{array}{c}\text { Post- } \\
\text { Intervention }\end{array}$ & $\begin{array}{c}\text { Pre- } \\
\text { Intervention }\end{array}$ & $\begin{array}{c}\text { Post- } \\
\text { Intervention }\end{array}$ \\
\hline Severe headache & 70.5 & 86.7 & 11.5 & 25.7 & 2.9 & 6.7 \\
\hline Blurred vision & 56.2 & 76.2 & 9.6 & 26.7 & 3.8 & 4.8 \\
\hline High BP & 58.1 & 59.0 & 32.7 & 28.6 & 25.7 & 15.4 \\
\hline PE/SPE & 24.8 & 45.7 & 2.9 & 17.1 & 1.0 & 12.5 \\
\hline Eclampsia or seizures & 65.7 & 70.5 & 58.7 & 77.1 & 50.5 & 64.4 \\
\hline Excessive vaginal bleeding & 71.4 & 66.7 & 90.4 & 95.2 & 91.4 & 84.6 \\
\hline High fever & 74.3 & 61.0 & 20.2 & 25.7 & 51.4 & 35.6 \\
\hline Tetanus & 2.9 & 1.9 & 1.0 & 2.9 & 11.4 & 4.8 \\
\hline Infant hand or feet first & - & - & 26.9 & 53.5 & - & - \\
\hline Prolonged labor & - & - & 77.9 & 74.3 & - & - \\
\hline Obstructed labor & - & - & 71.2 & 65.7 & - & - \\
\hline Retained placenta & - & - & 41.3 & 44.8 & - & - \\
\hline Ruptured uterus & - & - & 14.4 & 12.4 & - & - \\
\hline *Multiple responses & 105 & 105 & 104 & 105 & 105 & 105 \\
\hline
\end{tabular}

\section{Community service provider knowledge of PE/SPE/E signs and symptoms}

Community service providers' knowledge of specific PE/SPE/E signs and symptoms was also assessed before and after the intervention. Knowledge of high blood pressure (BP) as a sign of PE improved, from 60 percent at baseline to 91 percent post-intervention. Similarly, knowledge of albumin in urine, considered a sign of SPE in addition to high BP, increased from 29 percent to 82 percent (Table 4, following page). 
Analysis reveals that retention of $\mathrm{PE} / \mathrm{E}$ knowledge was similar, or higher, among $\mathrm{CHCPs}$ as it was for FWVs, which could be due to the fact recently recruited CHCPs are young, with better educational qualifications.

Table 4: Community facility-based provider knowledge of PE/SPE/E signs and symptoms*

\begin{tabular}{|c|c|c|}
\hline Signs and Symptoms & Pre-Intervention & Post-Intervention \\
\hline Pre-Eclampsia* & $\%$ & $\%$ \\
\hline High BP & 60.0 & 91.4 \\
\hline Excess albumin in the urine & 25.7 & 57.1 \\
\hline Headache & 0 & 27.6 \\
\hline Blurred vision & 0 & 21.9 \\
\hline Edema & 0 & 12.4 \\
\hline \multicolumn{3}{|l|}{ SPE* } \\
\hline Pregnancy-induced high BP & 63.8 & 94.3 \\
\hline Albumin in urine & 28.6 & 84.8 \\
\hline Severe headache & 60.0 & 67.6 \\
\hline Blurred vision/light sensitivity/seeing spots & 42.9 & 61.0 \\
\hline Upper abdominal pain (usually under ribs on the right side) & 8.6 & 51.4 \\
\hline Swelling (edema) particularly in face and hands & 57.1 & 46.7 \\
\hline \multicolumn{3}{|l|}{ Eclampsia* } \\
\hline Pregnancy-induced high BP plus & 59.0 & 67.3 \\
\hline Seizures/convulsions & 97.1 & 94.2 \\
\hline Albumin in urine plus & 24.8 & 54.8 \\
\hline *Multiple responses & 105 & 105 \\
\hline
\end{tabular}

\section{Community service provider knowledge of danger signs in pregnancy}

In addition to knowledge on PE/E, service providers were assessed on dangers signs during pregnancy. Table 5 shows that their knowledge increased substantially after the intervention period.

Table 5: Community facility-based provider knowledge of danger signs during pregnancy*

\begin{tabular}{|c|c|c|}
\hline Knowledge of Danger signs* & Pre-Intervention & Post-Intervention \\
\hline Seizures or convulsions & 88.6 & 94.3 \\
\hline Severe headache and blurred vision & 77.1 & 92.4 \\
\hline Vaginal bleeding & 91.4 & 89.5 \\
\hline High fever & 80.0 & 84.8 \\
\hline Prolonged labor & 55.2 & 66.7 \\
\hline Infant body parts other than head appear first during delivery & 0.0 & 4.8 \\
\hline Others & 10.5 & 1.0 \\
\hline *Multiple responses & 105 & 105 \\
\hline
\end{tabular}

\section{Pre- and post-intervention service statistics from intervention upazilas}

All ANC, delivery, PNC, and PE/E patients, as well as the total numbers of patients, were collected before and after the intervention, for adjacent nine month periods, from all community facilities in both upazilas. Service statistics were collected from DGFP's MIS reports. At baseline, service statistics were collected for January 2013 through September 2013, and collected for October 2013 through June 2014 at endline. These service statistics do not include figures from UHCs, MCWCs, or district hospitals (Table 6, following page). 
Table 6: Women who received services before and after the intervention, in 72 facilities

\begin{tabular}{|c|c|c|c|c|c|c|c|c|c|c|c|c|c|}
\hline \multicolumn{7}{|c|}{ Pre-Intervention } & \multicolumn{7}{|c|}{ Post-Intervention } \\
\hline Month & $\begin{array}{l}\text { General } \\
\text { Patients }\end{array}$ & ANC & PNC & Delivery & $\mathrm{PE} / \mathrm{E}$ & Total & Month & $\begin{array}{l}\text { General } \\
\text { Patients }\end{array}$ & ANC & PNC & Delivery & $\mathrm{PE} / \mathrm{E}$ & Total \\
\hline Jan'13 & 54,103 & 1,590 & 600 & 67 & 0 & 56,360 & Oct '13 & 53,025 & 1,406 & 411 & 50 & 0 & 54,892 \\
\hline Feb '13 & 53,332 & 1,404 & 618 & 43 & 0 & 55,397 & Nov'13 & 56,054 & 1,247 & 426 & 52 & 1 & 57,780 \\
\hline Mar'13 & 59,256 & 1,362 & 730 & 74 & 0 & 61,422 & Dec '13 & 56,542 & 1,400 & 523 & 57 & 2 & 58,524 \\
\hline Apr'13 & 63,648 & 1,465 & 726 & 109 & 0 & 65,948 & Jan '14 & 50,960 & 1,056 & 425 & 32 & 7 & 52,480 \\
\hline May '13 & 53,372 & 1,180 & 604 & 62 & 0 & 55,218 & Feb '14 & 49,194 & 1,040 & 378 & 34 & 6 & 50,652 \\
\hline Jun '13 & 30,131 & 916 & 472 & 57 & 0 & 31,576 & Mar '14 & 62,691 & 1,373 & 431 & 31 & 4 & 64,530 \\
\hline Jul'13 & 60,781 & 1,293 & 647 & 56 & 0 & 62,777 & Apr '14 & 52,192 & 1,368 & 453 & 42 & 5 & 54,060 \\
\hline Aug '13 & 60,204 & 1,243 & 666 & 56 & 0 & 62,169 & May '14 & 54,380 & 1,270 & 434 & 43 & 4 & 56,131 \\
\hline Sep '13 & 63,561 & 1,336 & 490 & 48 & 0 & 65,435 & Jun '14 & 54,380 & 1,269 & 435 & 44 & 4 & 56,132 \\
\hline Total (n) & 498,388 & 11,789 & 5,553 & 572 & 0 & 516,302 & Total (n) & 489,418 & 11,429 & 3,916 & 385 & 33 & 505,181 \\
\hline$\%$ & 96.5 & 2.3 & 1.1 & 0.1 & 0.0 & 100.0 & $\%$ & 96.9 & 2.3 & 0.8 & 0.1 & 0.007 & 100.0 \\
\hline
\end{tabular}

Analysis of service statistics suggests that numbers of patients, for ANC, delivery, and PNC services, did not change much over the course of the intervention. During the first three months of the intervention there was political unrest in the country, which may have decreased numbers of patients seeking services. Before the intervention period there were no documented $\mathrm{PE} / \mathrm{E}$ patients in the project areas, but during the intervention period 33 SPE/E patients presented for PE/E services.

\section{$\mathrm{PE} / \mathrm{E}$ patients treated in Brahmanbaria district}

To understand the magnitude of PE/E in the Brahmanbaria district, information was collected from various facilities. P/E service statistics were collected from the district Hospital, MCWC, UHCs, as well as private clinics and hospitals in the district; only statistics of PE/E patients treated during the intervention period were collected.

A total of 182 SPE/E patients were treated in Brahmanbaria district during the nine month intervention. Among the $182 \mathrm{PE} / \mathrm{E}$ patients, 51 patients sought treatment directly from the district hospital, 98 patients sought treatment directly at private clinics or hospitals, and 33 patients sought treatment from two intervention upazila UH\&FWCs-local community health care facilities. PE/E patients treated at these intervention facilities received a loading dose of $\mathrm{MgSO}_{4} \mathrm{IM}$ injection and were referred to the district hospital. Among these $33 \mathrm{SPE} / \mathrm{E}$ patients, one patient who received loading dose of $\mathrm{MgSO}_{4} \mathrm{IM}$ injection and referred to the district hospital instead went to a private health facility. District hospitals provided referral services for 84 SPE/E patients. No patient was treated at the MCWC, UHCs, or CCs (Table 7).

Table 7: PE/E patients treated in Brahmanbaria district, by facility type, during intervention

\begin{tabular}{|c|c|l|}
\hline Treatment Facility & Clients $(\mathrm{n})$ & \multicolumn{1}{c|}{ Remarks } \\
\hline District Hospital & 51 & Directly sought treatment from district hospital \\
\hline MCWC & None & Not fully functional due to manpower shortage \\
\hline UHC & None & Refer patients to district hospital \\
\hline UH\&FWC & 33 & $\begin{array}{l}\text { Patient sought treatment due to intervention, referred after } \text { MgSO }_{4} \text { loading dose } \\
\text { by community provider, treated at district hospital; one patient went to private } \\
\text { facility for treatment. }\end{array}$ \\
\hline CC & None & No patients went to a CC; clients prefer district hospital or private clinic care \\
\hline Private Clinic/Hospital & 98 & Directly sought treatment from private health facilities \\
\hline Total in 11 upazilas & 182 & Statistics are for 9 months, with no patients found during the initial 3 months \\
\hline
\end{tabular}




\section{$\mathrm{PE} / \mathrm{E}$ patients treated in the intervention area, by facility}

All SPE/E patients were treated by FWVs at UH\&FWCs. Although SACMOs, MAs, CHCPs, and CSBAs were trained and included in service provision, no SPE/E patients werer attended by them, and no patient went to them for service, indicating that FWVs are the most opportune community facility staff to provider a loading dose of $\mathrm{MgSO}_{4}$. More than 40 percent of SPE/E patients were treated at Sadar upazila's UH\&FWCs, which are generally more proximate than UHCs. In-depth interviews (IDIs) with Sadar upazila UH\&FWC service providers also indicate that their FWVs could be more confident due to ready UHC assistance in detecting and managing SPE/E, as the UHC has at least one doctor on duty at all times.

\begin{tabular}{|c|c|c|c|c|c|}
\hline Facility of Case Management & Pregnancy Duration & $\begin{array}{c}\text { BP } \\
(\mathrm{mm} \text { of } \mathrm{Hg})\end{array}$ & $\begin{array}{l}\text { Provider } \\
\text { Diagnosis }\end{array}$ & $\begin{array}{c}\text { Went to District } \\
\text { Hospital }\end{array}$ & Outcome \\
\hline \multicolumn{6}{|l|}{ Sarail upazila sub-district } \\
\hline Paniswar UH\&FWC & 39 weeks & $200 / 110$ & $\mathrm{E}$ & $\mathbf{Y}$ & Both Survived \\
\hline Sarail Sadar UH\&FWC & 40 weeks & $210 / 120$ & $E$ & $\mathrm{Y}$ & Both Survived \\
\hline Sarail Sadar UH\&FWC & 39 weeks & $160 / 120$ & $E$ & $\mathbf{Y}$ & Both Survived \\
\hline Sarail Sadar UH\&FWC & 39 weeks & $180 / 120$ & $\mathrm{E}$ & $\mathbf{Y}$ & Both Survived \\
\hline Sarail Sadar UH\&FWC & 40 weeks & $150 / 90$ & $\mathbf{E}$ & $\mathbf{Y}$ & Both Survived \\
\hline Sarail Sadar UH\&FWC & 41 weeks & $150 / 110$ & SPE & $\mathbf{Y}$ & Both Survived \\
\hline Shahazadapur UH\&FWC & 37 weeks & $190 / 120$ & $\mathbf{E}$ & $\mathbf{Y}$ & Both Survived \\
\hline Shahazadapur UH\&FWC & NI & $220 / 110$ & $\mathbf{E}$ & $\mathbf{Y}$ & Both Survived \\
\hline Shahazadapur UH\&FWC & 37 weeks & $150 / 100$ & $\mathbf{E}$ & $\mathbf{Y}$ & Both Survived \\
\hline Shahazadapur UH\&FWC & NI & $200 / 100$ & $E$ & $\mathbf{Y}$ & Both Survived \\
\hline Aruail UH\&FWC & $\mathrm{NI}$ & $150 / 100$ & SPE & $\mathbf{Y}$ & Both Survived \\
\hline Aruail UH\&FWC & 38 weeks & $160 / 100$ & $E$ & $\mathbf{Y}$ & Both Survived \\
\hline Noagaon UH\&FWC & 39 weeks & $140 / 100$ & $\mathbf{E}$ & $\mathbf{Y}$ & Both Survived \\
\hline Noagaon UH\&FWC & 39 weeks & $160 / 110$ & SPE & $\mathbf{Y}$ & Both Survived \\
\hline Noagaon UH\&FWC & $\mathrm{NI}$ & $160 / 100$ & $\mathrm{E}$ & $\mathbf{Y}$ & Both Survived \\
\hline Subtotal & & & $\begin{array}{c}15 \\
\text { (3 SPE, } 12 \mathrm{E})\end{array}$ & & All Survived \\
\hline \multicolumn{6}{|l|}{ Kasba upazila sub-district } \\
\hline Kasba Sadar UH\&FWC & 40 weeks & $180 / 100$ & SPE & $\mathbf{Y}$ & Both Survived \\
\hline Kasba Sadar UH\&FWC & $\mathrm{NI}$ & $150 / 100$ & $\mathbf{E}$ & $\mathbf{Y}$ & Both Survived \\
\hline Kasba Sadar UH\&FWC & 41 weeks & $150 / 100$ & SPE & $\mathbf{N}$ & Both Survived \\
\hline Kasba Sadar UH\&FWC & $\mathrm{NI}$ & $140 / 100$ & SPE & $\mathbf{Y}$ & Both Survived \\
\hline Kasba Sadar UH\&FWC & 40 weeks & $150 / 100$ & $\mathbf{E}$ & $\mathrm{Y}$ & Both Survived \\
\hline Kasba Sadar UH\&FWC & 28 weeks & $180 / 120$ & $E$ & $\mathbf{Y}$ & Both Survived \\
\hline Kasba Sadar UH\&FWC & $\mathrm{NI}$ & $180 / 100$ & $\mathbf{E}$ & $\mathbf{Y}$ & Both Survived \\
\hline Kasba Sadar UH\&FWC & NI & $140 / 90$ & $E$ & $\mathrm{Y}$ & Both Survived \\
\hline Kasba Sadar UH\&FWC & $\mathrm{NI}$ & $190 / 110$ & $E$ & $\mathrm{NI}$ & NI \\
\hline Kaimpur UH\&FWC & 41 weeks & $140 / 90$ & $\mathrm{E}$ & $\mathbf{Y}$ & Both Survived \\
\hline Kaimpur UH\&FWC & 39 weeks & $180 / 90$ & $\mathrm{E}$ & $\mathbf{Y}$ & Both Survived \\
\hline Bayek UH\&FWC & $\mathrm{NI}$ & $190 / 120$ & $\mathbf{E}$ & $\mathbf{Y}$ & Both Survived \\
\hline Bayek UH\&FWC & NI & $160 / 120$ & $\mathrm{E}$ & $\mathbf{Y}$ & Both Survived \\
\hline Mehari UH\&FWC & $\mathrm{NI}$ & $220 / 120$ & $\mathbf{E}$ & $\mathbf{Y}$ & Infant Died \\
\hline Mehari UH\&FWC & NI & $140 / 90$ & $\mathrm{E}$ & $\mathrm{Y}$ & Both Survived \\
\hline Binauti UH\&FWC & $\mathrm{NI}$ & $160 / 100$ & SPE & $\mathbf{Y}$ & Both Survived \\
\hline Binauti UH\&FWC & 40 weeks & $150 / 100$ & SPE & $\mathbf{Y}$ & Both Survived \\
\hline Binauti UH\&FWC & 41 weeks & $180 / 90$ & SPE & $\mathbf{Y}$ & Both Survived \\
\hline Subtotal & & & $\begin{array}{c}18 \\
(6 \mathrm{SPE}, 12 \mathrm{E})\end{array}$ & & $\begin{array}{c}\text { All Survived } \\
\text { except one infant }\end{array}$ \\
\hline Total & & & $\begin{array}{c}33 \\
\text { (9 SPE, } 24 \text { E) }\end{array}$ & & $\begin{array}{c}\text { All survived } \\
\text { except one infant }\end{array}$ \\
\hline
\end{tabular}


Findings suggest that most SPE/E patients treated only sought services during advanced stages of their conditions, indicating that when women do not seek services until complications are severe. Among the 33 patients treated in local public facilities, nine presented with SPE and the rest suffered seizures. Except one, all patients went to the district hospital for further management after referral.

Table 8 also indicates that 10 out of 19 UH\&FWCs in Sarail and Kasba sub-districts (5 UH\&FWCs in each upazila) saw PE/E patients, while the remaining nine saw none. No PE/E patients went to a CC, indicating service providers at these facilities were unable to implement the protocol, and indicates that CCs may not be optimal venues for implementation of a protocol for $\mathrm{MgSO}_{4} \mathrm{IM}$ injection loading doses. IDIs with service providers (all 10) indicate that community members do not yet believe local community service providers can effectively manage such complications. Observations reveal that many community health service providers are not at their offices with strict regularity, which may affect patient perceptions and attendance.

Outcomes of patient referrals were collected from the district hospital. No maternal or neonatal deaths occurred among those who received a $\mathrm{MgSO}_{4} \mathrm{IM}$ injection at a community facility-although one infant died at the hospital. No serious adverse effects due to loading dose of $\mathrm{MgSO}_{4} \mathrm{IM}$ were reported among the study population (Table 8). Two other infant deaths among PE/E patients occurred at the district hospital, after exclusive treatment from the district hospital, not referred from intervention upazilas.

\section{Socio-demographic characteristics of ANC clients}

To fully understand ANC in the district, exit client interviews assessed patients' characteristics and the quality of their ANC services. Findings reveal mean ages of ANC clients at baseline and endline of 24 and 26 , respectively. Before the intervention, about one fourth of respondents were younger than 19 , and after the intervention the proportion fell to one sixth. Approximately 10 percent ANC clients were illiterate preintervention, while 15 percent were illiterate during the intervention.

Table 9: Socio-demographic characteristics of ANC Clients

\begin{tabular}{|c|c|c|}
\hline Background Characteristic & Pre-Intervention, \% & Post-Intervention, \% \\
\hline \multicolumn{3}{|l|}{ Age } \\
\hline 19 and younger & 25.0 & 15.4 \\
\hline $20-24$ & 27.5 & 20.5 \\
\hline $25-29$ & 30.0 & 35.9 \\
\hline $30-34$ & 12.5 & 17.9 \\
\hline $35+$ & 5.0 & 10.3 \\
\hline Mean age & 23.95 & 25.90 \\
\hline $\mathrm{n}$ & 40 & 39 \\
\hline \multicolumn{3}{|l|}{ Education } \\
\hline None & 10.0 & 15.4 \\
\hline Primary, incomplete & 15.0 & 5.1 \\
\hline Primary, complete & 20.0 & 25.6 \\
\hline Secondary, incomplete & 40.0 & 28.2 \\
\hline Secondary, complete and higher & 15.0 & 25.6 \\
\hline Mean & 6.38 & 6.37 \\
\hline $\mathrm{n}$ & 40 & 38 \\
\hline \multicolumn{3}{|l|}{ Number of Children } \\
\hline 0 (first pregnancy) & 37.5 & 21.6 \\
\hline 1 & 22.5 & 24.3 \\
\hline 2 & 15.0 & 29.7 \\
\hline 3 and higher & 25.0 & 24.3 \\
\hline Mean number of children & 1.40 & 1.70 \\
\hline $\mathrm{n}$ & 40 & 37 \\
\hline
\end{tabular}


ANC clients' mean numbers of children were 1.4 pre-intervention and 1.7 during the intervention. Thirtyeight percent of women before the intervention, and 22 percent during the intervention, were primigravida. About one fourth of ANC clients had three or more children before and during the intervention.

\section{Antenatal care clients}

ANC clients were asked about their stage of pregnancy and reasons for their visit during client exit interviews. About three fifths of respondents before the intervention, and about half during the intervention, sought services in their third trimester. Twenty-eight out of 40 ANC clients before the intervention, and 29 (out of 39) during, came for routine checks without any unusual symptoms, while the remaining 12 clients before the intervention and 10 during sought routine checks as well as care for some sort of complication.

The ANC patients' reported problems did not differ significantly over time. Nine of the 12 ANC clients prior to the intervention, and eight of the 10 during the intervention, sought care for lower abdominal pain. Two clients in each period sought treatment for headache. One client before the intervention and two during had blurred vision. Because service providers do not write diagnoses on patient prescriptions or registers, some of those issues could have been related to PE/E (Table 10).

\section{Table 10: ANC clients, by pregnancy status (duration) and reason for current visit}

\begin{tabular}{|c|c|c|}
\hline Pregnancy Status and Reasons for ANC Visit & Pre-Intervention (n) & Post-Intervention (n) \\
\hline \multicolumn{3}{|l|}{ Month of Current Pregnancy } \\
\hline 6 months or fewer & 16 & 21 \\
\hline 7 to 8 months & 19 & 17 \\
\hline 9 months & 5 & 1 \\
\hline $\mathrm{n}$ & 40 & 39 \\
\hline \multicolumn{3}{|l|}{ Reason for Current ANC Visit } \\
\hline Routine check up & 28 & 29 \\
\hline With Problem & 8 & 3 \\
\hline Both & 4 & 7 \\
\hline $\mathrm{n}$ & 40 & 39 \\
\hline \multicolumn{3}{|l|}{ Problems* } \\
\hline Lower abdomen pain & 9 & 8 \\
\hline Weakness/vertigo & 4 & 2 \\
\hline Severe headache & 2 & 2 \\
\hline Blurred vision & 1 & 2 \\
\hline Swelling hand/feet/pre-eclampsia & 1 & - \\
\hline Foul smelling discharge with fever & - & 2 \\
\hline Others & 3 & 3 \\
\hline *Multiple responses & 12 & 10 \\
\hline
\end{tabular}

\section{Client and provider interactions}

Observations of client and provider interactions during ANC consultations focused on service provider practice and behaviors with ANC clients including history-taking, physical checks including BP measurement, in addition to information solicited from patients. With permission both provider and client, a trained female research assistant observed sessions of service provision. On average, two clients per provider were observed.

Analyses of client and provider observation data provide a poor and discouraging picture of ANC consultations, both before and during the intervention. Before the intervention, all service providers asked ANC clients about their last menstrual period, which decreased to 82 percent during the intervention. Similarly, before the intervention 12 out of 34 FWVs-but only three out of 25 during the intervention- 
asked about the patient's last delivery, whether normal or caesarean. Analysis of history-taking for essential care elements reveals it was poor among all service providers except FVWs (Table 11). Service provider training needs to be improved to explicitly cover systematic history-taking of ANC, delivery and PNC clients.

Table 11: Client and provider interactions

\begin{tabular}{|c|c|c|c|c|c|c|c|c|c|}
\hline \multirow[b]{2}{*}{ Provider Checked for and Informed Patient } & \multicolumn{5}{|c|}{ Pre-Intervention (n) } & \multicolumn{4}{|c|}{ Intervention (n) } \\
\hline & $\begin{array}{l}\text { FWV } \\
\text { Client }\end{array}$ & $\begin{array}{c}\text { SACMO } \\
\text { Client }\end{array}$ & $\begin{array}{l}\text { CSBA } \\
\text { Client }\end{array}$ & $\begin{array}{l}\text { CHCP } \\
\text { Client }\end{array}$ & Total & $\begin{array}{l}\text { FWV } \\
\text { Client }\end{array}$ & $\begin{array}{l}\text { CSBA } \\
\text { Client }\end{array}$ & $\begin{array}{l}\text { CHCP } \\
\text { Client }\end{array}$ & Total \\
\hline \multicolumn{10}{|l|}{ Patient History } \\
\hline Last menstrual period (LMP) & 34 & 2 & 2 & 2 & 40 & 21 & 4 & 7 & 32 \\
\hline Duration of gestation & 32 & 2 & 1 & 2 & 37 & 20 & 4 & 9 & 33 \\
\hline Type of last delivery (normal or caesarean) & 12 & 1 & 0 & 0 & 13 & 3 & 0 & 1 & 4 \\
\hline Place of last delivery & 9 & 0 & 0 & 0 & 9 & 3 & 0 & 1 & 4 \\
\hline Date of last delivery & 8 & 0 & 0 & 1 & 9 & 1 & 0 & 1 & 2 \\
\hline Duration of last labor & 1 & 0 & 0 & 0 & 1 & 1 & 0 & 1 & 2 \\
\hline Neonatal deaths & 3 & 0 & 0 & 0 & 3 & 6 & 0 & 0 & 6 \\
\hline Previous miscarriage or abortion & 13 & 0 & 1 & 0 & 14 & 1 & 0 & 0 & 1 \\
\hline Previous stillbirth & 6 & 0 & 0 & 0 & 6 & 6 & 0 & 0 & 6 \\
\hline History of hypertension & 3 & 0 & 1 & 0 & 4 & 6 & 0 & 1 & 7 \\
\hline History of diabetes & 0 & 0 & 0 & 0 & 0 & 0 & 0 & 0 & 0 \\
\hline History of asthma & 3 & 0 & 0 & 0 & 3 & 0 & 0 & 0 & 0 \\
\hline Current medication & 5 & 2 & 0 & 1 & 8 & 11 & 1 & 6 & 18 \\
\hline \multicolumn{10}{|l|}{ Physical Examination } \\
\hline Weight & 32 & 1 & 2 & 1 & 36 & 21 & 4 & 6 & 31 \\
\hline Height & 0 & 0 & 0 & 0 & 0 & 0 & 0 & 0 & 0 \\
\hline $\mathrm{BP}$ & 31 & 2 & 2 & 2 & 37 & 24 & 4 & 9 & 37 \\
\hline Pulse & 15 & 1 & 1 & 1 & 18 & 6 & 2 & 4 & 12 \\
\hline Eye for anemia & 33 & 2 & 1 & 1 & 37 & 17 & 4 & 9 & 30 \\
\hline Leg swelling & 28 & 1 & 1 & 1 & 31 & 11 & 3 & 5 & 19 \\
\hline Temperature & 3 & 1 & 0 & 1 & 5 & 0 & 0 & 1 & 1 \\
\hline Breast & 11 & 0 & 1 & 0 & 12 & 3 & 1 & 0 & 4 \\
\hline Checked abdomen for fetal position & 33 & 1 & 2 & 1 & 37 & 16 & 1 & 2 & 19 \\
\hline Checked fetal movement & 25 & 1 & 2 & 1 & 29 & 17 & 1 & 2 & 20 \\
\hline Checked fetal heart rate & 27 & 1 & 2 & 1 & 31 & 15 & 1 & 2 & 18 \\
\hline \multicolumn{10}{|l|}{ Provider Performed or Referred for Laboratory Testing } \\
\hline Urine test for pregnancy & 4 & 0 & 0 & 0 & 4 & 0 & 0 & 0 & 0 \\
\hline Hemoglobin test & 5 & 0 & 0 & 0 & 5 & 0 & 0 & 0 & 0 \\
\hline Ultra-sonogram & 9 & 0 & 0 & 0 & 9 & 0 & 0 & 0 & 0 \\
\hline Syphilis test & 0 & 0 & 0 & 0 & 0 & 1 & 0 & 0 & 1 \\
\hline Urine albumin test & 0 & 0 & 0 & 0 & 0 & 7 & 0 & 0 & 7 \\
\hline Recorded all information in register, ANC card & 31 & 2 & 1 & 2 & 36 & 15 & 2 & 7 & 24 \\
\hline Medicine to prevent anemia & 34 & 2 & 2 & 2 & 40 & 25 & 4 & 10 & 39 \\
\hline \multicolumn{10}{|l|}{ Provider Informed, Discussed, Advised } \\
\hline Informed of where to go for complication & 18 & 0 & 0 & 1 & 19 & 5 & 0 & 2 & 7 \\
\hline High fever a danger sign during pregnancy & 11 & 1 & 0 & 0 & 12 & 0 & 0 & 0 & 0 \\
\hline Blurred vision a danger sign during pregnancy & 10 & 0 & 0 & 1 & 11 & 8 & 0 & 2 & 10 \\
\hline Severe headache a danger sign when pregnant & 10 & 0 & 0 & 1 & 11 & 2 & 0 & 0 & 2 \\
\hline Excessive bleeding a pregnancy danger sign & 4 & 0 & 0 & 0 & 4 & 2 & 0 & 1 & 3 \\
\hline Labor pains of than 12 hours a danger sign & 5 & 0 & 0 & 0 & 5 & 1 & 0 & 0 & 1 \\
\hline Seizures (eclampsia) are danger signs & 6 & 0 & 0 & 0 & 6 & 0 & 0 & 0 & 0 \\
\hline Informed about expected delivery date (EDD) & 32 & 2 & 2 & 2 & 38 & 17 & 4 & 6 & 27 \\
\hline Informed about TT injection & 18 & 0 & 1 & 0 & 19 & 4 & 3 & 2 & 9 \\
\hline Food quantity/quality during/after pregnancy & 30 & 2 & 1 & 2 & 35 & 21 & 4 & 8 & 33 \\
\hline
\end{tabular}




\begin{tabular}{|c|c|c|c|c|c|c|c|c|c|}
\hline Personal hygiene & 20 & 2 & 0 & 2 & 24 & 13 & 4 & 7 & 24 \\
\hline Importance of FP after delivery & 6 & 0 & 1 & 0 & 7 & 1 & 0 & 0 & 1 \\
\hline When to start FP method & 2 & 1 & 0 & 0 & 3 & 1 & 0 & 0 & 1 \\
\hline \multicolumn{10}{|l|}{ Provider Discussed Birth Planning } \\
\hline Sterilizing supplies for home delivery & 12 & 0 & 0 & 0 & 12 & 2 & 0 & 1 & 3 \\
\hline Arranging blood donor or blood supply & 5 & 0 & 0 & 0 & 5 & 0 & 0 & 1 & 1 \\
\hline Saving money & 12 & 0 & 0 & 1 & 13 & 0 & 0 & 2 & 2 \\
\hline Arranging vehicle & 1 & 0 & 0 & 0 & 1 & 0 & 0 & 1 & 1 \\
\hline Arranging attendant to accompany at hospital & 1 & 0 & 0 & 0 & 1 & 0 & 0 & 0 & 0 \\
\hline Select a facility or SBA for delivery & 20 & 0 & 2 & 1 & 23 & 3 & 1 & 2 & 6 \\
\hline$n$ & 34 & 2 & 2 & 2 & 40 & 25 & 4 & 10 & 39 \\
\hline
\end{tabular}

Physical examination is vitally important during ANC. Most providers weigh ANC clients and measure BP; few performed or referred ANC clients for laboratory testing. ANC information or advice on essential matters was also poor. FWVs were more likely to inform their clients that blurred vision is a danger sign during ANC period, however. Similarly, birth planning was poorly discussed with ANC clients by all service providers except FWVs, and only about one third of those providers discussed birth planning prior to the intervention, and few did during the post-intervention period (Table 11).

\section{Service provider opinions of PE/E training}

Service providers from community facilities were asked during the post-intervention survey about their training in $\mathrm{PE} / \mathrm{E}$ management for this study. Less than half of service providers believed their training was adequate, with most stating that one week of training was necessary. About 12 percent of surveyed providers suggested refresher training, and another 10 percent requested more practical sessions, particularly CSBA and CHCP (Table 12).

Table 12: Distribution of service providers by views/opinions on PE/E management training

\begin{tabular}{|c|c|c|c|c|c|c|}
\hline \multirow{2}{*}{ PE/E Training Sufficient? } & \multicolumn{5}{|c|}{ Provider (n) } & \multirow{2}{*}{ Total } \\
\hline & FWV & SACMO & Nurse & CSBA & CHCP & \\
\hline Yes & 11 & 5 & 1 & 15 & 14 & $46(43.8 \%)$ \\
\hline No & 8 & 6 & 1 & 23 & 21 & $59(56.2 \%)$ \\
\hline$n$ & 19 & 11 & 2 & 38 & 35 & 105 \\
\hline \multicolumn{7}{|l|}{ Training aspects where need improvement } \\
\hline Training Elements & FWV & SACMO & Nurse & CSBA & CHCP & Total \\
\hline One week of training needed & 7 & 5 & 1 & 16 & 16 & $45(76.3 \%)$ \\
\hline Refresher training needed & 0 & 1 & 0 & 3 & 3 & 7 (11.9\%) \\
\hline More practical sessions needed & 1 & 0 & 0 & 4 & 2 & $6(10.1 \%)$ \\
\hline$n$ & 8 & 6 & 1 & 23 & 21 & 59 \\
\hline
\end{tabular}

\section{Program manager opinions of PE/E management training}

The post-intervention survey also asked referral facility program managers and their service providers about the PE/E training of community facility service providers. Program managers and service providers at referral facilities were positive about the trainings, and all $(n=6)$ said that the training program improved community service providers' knowledge and efficiency in detecting and managing PE/E and maternal health issues. They also cited field workers' efforts in raising community PE/E awareness. 


\section{Service provider assessments of PE/E patient management after training}

During training, service providers demonstrated skills in measuring BP, detecting albumin in urine, and administering $\mathrm{MgSO}_{4}$ into a dummy figure. It was not possible, though, to observe IM administration due to the impossibility of predicting SPE/E patient, which thus would require 24 hour project surveillance of community facilities. At endline, service providers were asked about their abilities in measuring BP and albumin in urine, and administering loading doses of $\mathrm{MgSO}_{4} \mathrm{IM}$ injection in two buttocks.

All interviewed community service providers said they could measure BP, except a few-one FWV, one SACMO, one nurse, and two CHCPs-who were unsure. Several service providers-three FWVs, five SACMOs, one nurse, 12 CSBAs, and six CHCPs-said they were unable or uncertain how to test and detect albumin in urine. A number of service providers-four FWVs, four SACMOs, one nurse, 17 CSBAs, and 15 CHCPssaid they were unable or not sure how to administer a loading dose of $\mathrm{MgSO}_{4}$ after their training (Table 13).

Only about half of FWVs could gauge their abilities through actual PE/E patient management, as all other community service providers had no PE/E patients, and could not put their training into practice.

Table 13: Service provider self-assessments of skills in PE/E patient management after training

\begin{tabular}{|c|c|c|c|c|c|c|c|c|c|c|c|c|}
\hline \multirow[t]{2}{*}{ Provider } & \multicolumn{4}{|c|}{ Able to Measure BP Efficiently ( $n$ ) } & \multicolumn{4}{|c|}{$\begin{array}{l}\text { Able to Test and Detect Albumin } \\
\text { in Urine (n) }\end{array}$} & \multicolumn{4}{|c|}{$\begin{array}{l}\text { Able to Administer } \mathrm{MgSO}_{4} \text { IM } \\
\text { Efficiently }(\mathrm{n})\end{array}$} \\
\hline & Yes & No & Unsure & Total & Yes & No & Unsure & Total & Yes & No & Unsure & Total \\
\hline FWV & 18 & 0 & 1 & 19 & 16 & 2 & 1 & 19 & 15 & 3 & 1 & 19 \\
\hline SACMO & 10 & 0 & 1 & 11 & 6 & 4 & 1 & 11 & 7 & 3 & 1 & 11 \\
\hline Nurse & 1 & 0 & 1 & 2 & 1 & 0 & 1 & 2 & 1 & 0 & 1 & 2 \\
\hline CSBA & 38 & 0 & 0 & 38 & 26 & 12 & 0 & 38 & 21 & 17 & 0 & 38 \\
\hline $\mathrm{CHCP}$ & 33 & 0 & 2 & 35 & 29 & 4 & 2 & 35 & 20 & 13 & 2 & 35 \\
\hline Total, $\mathrm{n}$ & 100 & 0 & 5 & 105 & 78 & 22 & 5 & 105 & 64 & 36 & 5 & 105 \\
\hline$\%$ & 95.2 & 0 & 4.8 & 100 & 74.2 & 21.0 & 4.8 & 100 & 61.0 & 34.2 & 4.8 & 100 \\
\hline
\end{tabular}

\section{Community service provider opinions of $\mathrm{MgSO}_{4}$ loading dose administration at community health care facilities}

Almost all service providers were in favor of administering of a loading dose of $\mathrm{MgSO}_{4} \mathrm{IM}$ injection at community facilities (Table 14).

Table 14: Opinions of $\mathrm{MgSO}_{4}$ loading dose administration at community facilities

\begin{tabular}{|c|c|c|c|c|}
\hline \multirow[b]{2}{*}{ Provider } & \multicolumn{3}{|c|}{ Should the loading dose of $\mathrm{MgSO}_{4}$ be given at community facilities, $\mathrm{n}(\%)$} & \multirow{2}{*}{$\mathbf{n}$} \\
\hline & Yes & No & Not Sure & \\
\hline FWV & $17(89.5)$ & $1(5.3)$ & $1(5.3)$ & 19 \\
\hline SACMO & $10(90.9)$ & 0.0 & $1(9.1)$ & 11 \\
\hline Nurse & $1(50.0)$ & 0.0 & $1(50.0)$ & 2 \\
\hline CSBA & $38(100.0)$ & 0.0 & 0.0 & 38 \\
\hline CHCP & $33(94.3)$ & 0.0 & $2(5.7)$ & 35 \\
\hline Total, $\mathrm{n}$ & 99 & 1 & 5 & 105 \\
\hline$\%$ & 94.3 & 1.0 & 4.8 & 100 \\
\hline
\end{tabular}

\section{Client choice of district hospitals for SPE/E management}

Community service providers were asked about client preferences for district hospitals for SPE/E management, with three primary reasons: 1 ) easy transportation to district hospitals $(81.8 \%)$, 2) seeking specialist care $(71.7 \%)$, and 3 ) lack of confidence in community health care facilities (Table 15, next page). 
Table 15: Reasons for client choice of district hospital

\begin{tabular}{|l|c|}
\hline & Number (\%) \\
\hline Easy transportation system to district hospital & $\mathbf{8 1 ( 8 1 . 8 )}$ \\
\hline Awareness of the people to seek specialist care & $71(71.7)$ \\
\hline Not confident about the community level facilities & $39(39.4)$ \\
\hline Lack of knowledge about the availability of PE/E service & $\mathbf{8 ( 8 . 1 )}$ \\
\hline Activity of the agents of different private facilities & $7(7.1)$ \\
\hline & $\mathrm{n}$ \\
\hline
\end{tabular}

District, upazila, and sub-district service providers and program managers were also asked about client choice of district hospitals instead of community facilities for PE/E management. The primary reasons offered by respondents $(n=6)$, during the post-intervention survey, were:

- Lack of availability of community service providers in local facilities

- Perception about the ability of service provider to manage PE/E patients at their expected level, and

- Lack of confidence in community service providers in emergency conditions like eclampsia.

These findings indicate that more efforts are required to make community members not only aware of, but confident in utilizing, the services provided at their local health facilities. Service providers and program managers suggested regular trainings for community service providers on maternal health, PE/E detection and referral, in addition to building community awareness of services available at community facilities.

\section{Program manager and referral service provider opinions of implementation}

The baseline survey conducted 14 IDIs with program managers and referral facility service providers, who were asked whether they received any referred SPE/E patients. Doctors from Sarail and Kasba upazila UHCs (8 out of 14) reported that their facilities received SPE/E patients, but they referred patients to the district hospital, as they were not equipped to deal with such patients.

During the post-intervention survey, six program managers and referral service providers were interviewed about administration of $\mathrm{MgSO}_{4} \mathrm{IM}$ injection loading dose at community facilities, in addition to program expansion. Specific questions were: 1 ) whether $\mathrm{MgSO}_{4}$ loading dose should be administered at community facilities, 2) whether community facility-based service providers are willing to administer $\mathrm{MgSO}_{4} \mathrm{IM}$ injection, and 3) whether loading dose protocol should be expanded to other parts of the country.

A majority of respondents (5 out of 6 ) stated that the program should be expanded to other areas of the country. All program managers and referral service providers $(n=6)$ are in favor of its expansion. One program manager stated, "This program improved the efficiency of the service provision of community service providers, their knowledge on maternal health related complications, detection and management of PE/E has increased and helped in building awareness among community people about maternal health." It was also mentioned that it will be difficult to implement the program efficiently due to the existing workloads of community service providers.

\section{Referrals}

Referrals from community facility service providers to referral facilities worked well, although community service providers did not use the referral slips; referral facility staff provided the requested treatments, regardless. In a few cases, community service providers did consult referral facility service providers before serving SPE/E patients. 
This study makes it evident that the district hospital is the ideal place for patient referrals, where SPE/E patients can receive all services including caesarian section. District hospitals should be included in future programs for any improvements necessary for serving as referral facilities.

\section{Cost of the intervention and scaling up}

A discrete exercise assessed the cost for expanding the intervention within one upazila or sub-district, with yearly recurring costs. The cost for every item and event was collected pre-design. All research, capital, and opportunity costs borne by government staff were excluded from analysis.

The unit cost of the intervention per upazila or sub-district is US\$7,948, with annual recurring cost of US $\$ 2,052$. Refresher training creates additional cost of US $\$ 4,046$ per upazila. This analysis assumed FWVs to be the primary providers of $\mathrm{MgSO}_{4} \mathrm{IM}$ injection loading doses in communities during expansion. Other service providers such as SACMOs, MAs, CSBAs, CHCPs, HAs, and FWAs require orientation for detecting and referring PE/SPE/E clients to UH\&FWCs for further management.

Table 16: Intervention costs in two upazilas and recurring cost for program continuation, in taka

\begin{tabular}{|c|c|c|c|c|}
\hline \multirow{2}{*}{ Elements } & \multicolumn{3}{|c|}{ Intervention Costs in Two Upazilas } & \multirow{2}{*}{ Recurring Costs } \\
\hline & Quantity & Rate & Total & \\
\hline Training of Trainer cost $(2 \times 10000 \times 1$ day $)$ & 2 & 10,000 & 20,000 & \\
\hline \multicolumn{5}{|l|}{ District orientation $(20 \times 1000 \times 1$ day $)$} \\
\hline Per diem & 20 & 10,00 & 20,000 & \\
\hline Refreshment for participants & 20 & 300 & 6,000 & \\
\hline Trainer cost $(2 \times 5000 \times 3$ days $)$ & 6 & 5,000 & 30,000 & \\
\hline Trainer transportation cost (3 days) & 3 & 7,000 & 21,000 & \\
\hline \multicolumn{4}{|l|}{ Upazila orientation for field workers } & 149,500 \\
\hline Per diem & 180 & 500 & 90,000 & \\
\hline Refreshment & 180 & 300 & 54,000 & \\
\hline Venue charge ( 4 batches) & 4 & 10,000 & 40,000 & \\
\hline Trainer cost $(2 \times 5000 \times 5$ days $)$ & 10 & 5,000 & 50,000 & \\
\hline Accommodation & 10 & 3,000 & 30,000 & \\
\hline Trainer transportation cost (5 days) & 5 & 7,000 & 35,000 & \\
\hline \multicolumn{4}{|l|}{ Community service provider training } & 30,500 \\
\hline Per diem & 20 & 1,000 & 20,000 & \\
\hline Refreshment & 20 & 300 & 6,000 & \\
\hline Venue & 1 & 5,000 & 5,000 & \\
\hline Trainer cost $(2 \times 5000 \times 1$ day $)$ & 2 & 5,000 & 10,000 & \\
\hline Accommodation & 2 & 3,000 & 6,000 & \\
\hline Trainer transportation cost ( 2 days) & 2 & 7,000 & 14,000 & \\
\hline \multicolumn{4}{|l|}{ Other community service provider orientation } & 127,500 \\
\hline Per diem & 120 & 1,000 & 120,000 & \\
\hline Refreshment & 120 & 300 & 36,000 & \\
\hline Venue charge & 3 & 10,000 & 30,000 & \\
\hline Trainer cost $(2 \times 5000 \times 3$ days $)$ & 6 & 5,000 & 30,000 & \\
\hline Accommodation (3 days) & 6 & 3,000 & 18,000 & \\
\hline \multirow[t]{2}{*}{ Trainer transportation cost (3 days) } & 3 & 7,000 & 21,000 & \\
\hline & \multicolumn{3}{|c|}{$\begin{array}{l}\text { Taka upazila recurring cost (for refresher training) } \\
\text { US\$ upazila recurring cost (for refresher training) }\end{array}$} & $\begin{array}{c}307,500 \\
4,046\end{array}$ \\
\hline BCC materials & 1 & 115,500 & 115,500 & 57,750 \\
\hline Photocopies & 10,000 & 2 & 20,000 & \\
\hline Urine strip (60 box) & 60 & 300 & 18,000 & 9,000 \\
\hline
\end{tabular}




\begin{tabular}{|c|c|c|c|c|}
\hline BP machine (69 pcs) & 69 & 1,450 & 100,050 & \\
\hline Stethoscope (69 pcs) & 69 & 350 & 24,150 & \\
\hline $\mathrm{MgSO}_{4}$ injections & 3,800 & 31 & 117,800 & 58,900 \\
\hline Printing of national protocol & 400 & 100 & 40,000 & \\
\hline Monitoring costs-from central MoH\&FW & 6 & 10,100 & 60,600 & 30,300 \\
\hline Total Intervention Cost for Two Upazilas & & & $1,208,100$ & \\
\hline Total Intervention Cost for One Upazila & 1 upazila & & 604,050 & 155,950 \\
\hline One Upazila in US \$ & $@ 76$ & & 7,948 & 2,052 \\
\hline
\end{tabular}




\section{Discussion}

Eclampsia contributes to approximately 20 percent of maternal deaths in Bangladesh, and most of those deaths occur as a result of insufficient maternity care within local communities, due to ignorance of the condition, lack of ANC, and delays in reaching facilities for appropriate care. These deaths can be easily prevented using low cost maternal health commodities. If community health care facility staff are trained to detect $\mathrm{PE} / \mathrm{E}$, administer a loading dose of $\mathrm{MgSO}_{4}$, and refer diagnosed patients with sufficient time for appropriate case management at referral facilities, or by qualified physicians, perinatal mortality can be greatly reduced.

This study was conducted in operational government clinics without any major adjustments to standard operations and procedures. Government health care and FP service providers based at local community facilities, generally UH\&FWCs, implemented the protocol, with government field workers conducting awareness activities and identifying high risk pregnant women and then referring them to UH\&FWCs.

During the nine months of this study about half of UH\&FWCs treated SPE/E patients, ranging one to nine patients, but CCs, which are smaller, more recently established public clinics in local neighborhoods, had no SPE/E patients. This study could not assess whether SCAMOs, CSBAs, and CHCPs are able to detect $\mathrm{PE} / \mathrm{E}$, administer loading dose of $\mathrm{MgSO}_{4}$, and refer patients because none cared for PE/E patients, so it remains difficult to say whether these service provider cadres can provide PE/E services acceptably. As a result, CCs and their affiliated service providers may not be the optimal place for implementing a national protocol for administering a loading dose of $\mathrm{MgSO}_{4} \mathrm{IM}$ injection for PE/E patients.

FWVs who treated SPE/E patients showed competence in detecting PE/E patients, including urine testing, administering a loading dose of $\mathrm{MgSO}_{4}$ and referring patients with sufficient time for successful case management. FWVs should be the focus of implementation of the national protocol in the future. FWVs, SACMOs, and CSBAs are proved more capable than nurses and CHCPs in PE/E detection and $\mathrm{MgSO}_{4} \mathrm{IM}$ administration, but it is not advisable to include community health workers other than FWVs in the revised national protocol, as CHCPs are newly recruited and are stationed at CCs, SACMOs rarely handle ANC patients, and those patients rarely contact CCs, SACMOs, or even CSBAs for ANC services.

Because PE/E patients did not utilize many community health care facilities, many local service providers expostulated that they were unable to apply their newly achieved skills. Observation and analysis reveals that FWVs with regular attendance at their health facilities did care for SPE/E patients. Only about one third of FWVs and SACMOs reside at their facility residences, although all are supposed to.

Lack of regular availability of service providers negatively affects patient intentions to seek care from community facilities during emergencies. Program managers suggested similar reasons for low community health care facility utilization. Monitoring visits revealed that local facility infrastructure is favorable in most areas; it is perceived lack of commitment by community service providers and field workers that influence public opinion. Many are committed to other government health activities for other priority areas.

Field worker visits to pregnant women are minimal, and their abilities for mastering technical knowledge for identifying and detecting PE/E are questionable. Training them to identify PE/E signs and symptoms should, however, be part of their training and job expectations, along with raising community awareness about PE/E and referring clients to UH\&FWCs. CSBAs also do not have client confidence for PE/E detection and management.

Preference for specialist care of SPE/E is evinced by patients' primary utilization of district hospitals and private facilities, indicating lack of confidence in community facilities, or lack of awareness of available $\mathrm{PE} / \mathrm{E}$ services. Community facilities should re-assess and revise their service provision, starting with guaranteed, regular provider availability, so their communities gain confidence in their reliability for services 
whenever needed, with additional re-branding and complete dissemination of these reforms within their communities.

Service providers were inconsistent and unreliable in using project tools to record requisite patient information. They cited time constraints and number of other required registers for their lack of compliance. As a result, the project used their MIS reports for service statistics. A number of inconsistencies were found between their facility registers and reported statistics. During additional studies data collection tools need to be minimized, and health officials should audit the accuracy of their existing registers and MIS tools and make changes to improve their accuracy.

Observation of actual SPE/E service provision by community service providers was impossible due to the impossibility of determining PE/E client visits. The study relied on service providers' self-assessments of their administration of the loading dose of $\mathrm{MgSO}_{4} \mathrm{IM}$ injection. All service providers were trained in IM injection. The study used $\mathrm{MgSO}_{4}$ solution for IM injection, which is simple and safe. As the study did not use powder for solution, service providers had no problems related to mixing for actual amounts or doses. OGSB's experiences with $\mathrm{MgSO}_{4} \mathrm{IM}$ injection in national referral hospitals suggested that $\mathrm{MgSO}_{4}$ is quite safe, with side effects rare, and a known antidote is readily available.

Analyses of pre- and post-intervention data suggest that participants began with a lack of knowledge for many maternal health issues, and improved marginally due to training: Although knowledge improved in some respects, it was not sufficient. Significant improvements in community facility providers' knowledge of PE/E were observed, however. Community service providers' training was limited to PE/E detection, including physical examination, BP measurement, and urine test, condition management, $\mathrm{MgSO}_{4}, \mathrm{IM}$ injection loading dose administration, and patient referrals. Other maternal health issues such as lifethreatening complications during pregnancy, delivery, and PNC, except PE/E, were not given enough importance in training. Although the lack of improvement in general maternal health knowledge among community service providers could have been due to weak training or poor questioning by interviewers, who were more focused on PE/E issues, it is still recommended that service provider training includes broader maternal health issues.

More than two thirds of patients reported seizures. This complication may be due to the fact pregnant women do not seek treatment early enough, or that service providers failed to diagnose problems during ANC. About one third of SPE patients presented with variable BP ranges, proteinuria, and other signs and symptoms.

Follow up of the diagnosed and referred 33 patients in this study showed promising results, and no perinatal deaths were reported-with the exception of one infant death at referral care facility. This finding indicates that community facilities, particularly UH\&FWCs, should be promoted to detect and manage PE/E patients. As no patients went to CCs, or to the other cadres who sits in the facility, this facility and the service providers may be excluded from providing loading dose of $\mathrm{MgSO}_{4} \mathrm{IM}$ injection at the community.

Data from before and after the intervention on client and provider interactions present a poor picture of service provider practice. In multiple cases, service provider knowledge and practice declined postintervention. This again could be due to unreliable recording by observers and poor interviewing, but the training module for service providers should be reviewed to ensure that other aspects of maternal health, particularly pregnancy complications, delivery issues, postpartum hemorrhage, and PNC complications, are emphasized. Their job aids and service protocols should be reviewed, simplified, but strengthened.

Exit client interviews reinforce the dismal picture of client and provider interactions. Service providers were not consistent in information provision, physical examinations, and birth planning discussion. Either service providers do not practice all essential elements of ANC regularly, through negligence, or they had poor training. 
Service provider interviews and surveys, at least, indicate poor training as an issue of concern, as two thirds reported inadequate training. Findings indicate that community service providers based need training on broader reproductive and maternal health issues, history-taking, physical examination, diagnosis, and treatment. Training only in $\mathrm{PE} / \mathrm{E}$, which was provided for this intervention study, is inadequate for addressing the training needs of community service providers. Service provider practices in BP measurement and diagnoses did not change due to intervention training; documenting BP and diagnoses in patient registers was a challenge for study subjects.

Those community service providers who managed PE/E patients were enthusiastic about the program and thought the protocol and its services should be expanded to other areas. Program managers and referral service providers expressed similar opinions, but they also referenced many challenges including the workloads and existing commitments of service providers and field workers, along with community members' lack of confidence in community service providers during emergencies. They suggested more work in raising community awareness of services available at local community health care facilities.

\section{Challenges}

- Rural residents are not fully aware of not only the dangers of pre-eclampsia but its signs and symptoms. Some consider such symptoms as typical during pregnancy. Informing and educating community members, through field workers, presents challenges because most field workers do not visit homes and are overburdened with assigned tasks.

- Regular BP measurement and documentation of all ANC, delivery, and PNC clients, in addition to urine albumin testing, by community facility service providers is challenging.

- In some instances, patients or their companions refused a loading dose of $\mathrm{MgSO}_{4} \mathrm{IM}$ injection at community facilities due to their lack of confidence in community service providers, in addition to some difficulties encountered in convincing patient companions of the necessity of emergency management and referrals for SPE/E patients.

- Community health care facilities do not provide services at all hours, which deters people from seeking care, especially during emergencies. Staff absenteeism, which can often be due to patient care outside facilities, is also common, which further discourages people from seeking services at community facilities.

- The rate of pregnant women seeking ANC, delivery, and PNC at community facilities was low and did not increase due to the intervention. Standard or universal facility provision of ANC, delivery, and PNC will continue to be a health system challenge.

- Compliance in documenting relevant information about ANC, delivery, and PNC clients in facility registers was not uniform at all facilities. Motivating service providers to record complete patient information is a challenge, necessitating a frequent and strong monitoring and supervision system. 


\section{Conclusions and Recommendations}

- Several SPE/E patients presented at intervention UH\&FWCs, yet none visited CCs or CSBAs. All presenting eclamptic patients reported seizure as the primary complaint, while SPE patients reported variable high BP, proteinuria, and other signs or symptoms. The service providers who examined SPE/E patients administered a loading dose of $\mathrm{MgSO}_{4} \mathrm{IM}$ injection and referred each one with sufficient time for successful case management.

- Field workers did not refer any clients with signs and symptoms consistent with PE/E, and instead presenting patients were seeking emergency care. To make the program sustainable, field workers (FWAs and HAs) should be fully motivated and involved in raising PE/E awareness in their communities, detect $\mathrm{PE} / \mathrm{E}$ patients through signs and symptoms, and refer patients to the community facilities, if needed. Collaboration is needed between both MoH\&FW directorates to make the program successful.

- All participating service providers and program managers are enthusiastic about the intervention and suggested its expansion to other parts of the country. Intervention replication should be phased, however, initially in a larger area to fully understand operational complexities and any requisite adjustments, prior to implementation throughout the country. Satellite clinics are not appropriate for this intervention, particularly because of necessary urine testing, which requires a washroom.

- The unit cost per upazila or sub-district for this intervention's expansion is calculated at US\$7,948, with an annual recurring cost of US\$2,052. Refresher trainings require additional cost of US $\$ 4,046$ per upazila.

- Expansion of this intervention should prioritize and focus on FWVs, and possibly CSBAs, through the adoption of a new, or revised, national protocol, with greater simplification.

- Essential instruments and equipment such as BP machines and stethoscopes are either non-functioning or unavailable at community health care facilities. Test tubes for urine collection and testing and disposable syringes and needles are also not available. All of these materials are required for such an intervention.

- The preferred solution of $\mathrm{MgSO}_{4}$ for an IM injection loading dose is in one or two vials, rather than four. Communication and advocacy with manufactures to make this adjustment is an absolute necessity.

- Community service providers need mentorship, particularly at the beginning of the intervention. Each referral facility must designate a staff member as a resource person to make the referral mechanism effective, providing direct access to community facility service providers via mobile phone for assistance while needed. The CLFBSP educational curriculum should be revised to incorporate PE/E diagnosis and treatment.

- More than half of community service providers evaluated their training for this intervention as inadequate. Future training should include broader maternal health elements such as ANC, delivery, and PNC, and not exclusively PE/E. As service providers urge refresher training for themselves, it should be included in the intervention protocol, scheduled for providers after six months of implementation, to refresh their knowledge, share experiences of care, and offer solutions to challenges, if any.

- Strategies need to be adopted to ensure regular availability of services and service providers at community facilities. Monitoring and supervision are crucial in this regard.

- As community facilities do not command full confidence among their clientele, this perception must be addressed, starting with consistent service hours in facilities, followed by massive dissemination of their service availability, including PE/E services, within their catchment areas, with potential additional rebranding. 


\section{References}

1. World Health Organization. 2005. Make Every Mother and Child Count. The World Health Report 2005. Geneva: World Health Organization.

2. World Health Organization. 2011. WHO recommendations for prevention and treatment of preeclampsia and eclampsia. Geneva: World Health Organization.

3. National Institute of Population Research and Training, MEASURE Evaluation, ICDDR,B. 2012. Bangladesh Maternal Mortality and Health Care Survey 2010. Dhaka: National Institute of Population Research and Training, MEAUSRE Evaluation, and ICDDR,B.

4. Government of Bangladesh. 2007. Millennium Development Goals, Mid-term Bangladesh Progress Report 2007. Dhaka: Planning Commission, Government of Bangladesh.

5. Yasmin HA. Baseline Survey for assessment of Emergency Obstetric Care Services in Bangladesh. 1995. Dhaka: The Bangladesh Institute of Research for Promotion of Essential \& Reproductive Health and Technologies.

6. NIPORT, Mitra and Associates, Measure DHS+ ORC Macro Internationals Inc. 2011. Bangladesh Demographic and Health Survey. Dhaka: NIPORT, Mitra and Associates, Measure DHS+ ORC Macro Internationals Inc.

7. Eclampsia Trial Collaborative Group 1995. Which anticonvulsant for women with eclampsia? Evidence from the collaborative eclampsia trial. Lancet 345: 1455-1463.

8. Magpie Trial Collaborative Group 2002. Do women with pre-eclampsia and their babies benefit from magnesium sulphate? The magpie trial: a randomized placebo-controlled trial. Lancet 359: 18771890. 